\title{
P02-235
}

\section{REASONS FOR CHOICE OF MEDICATION - BASELINE DATA OF THE OUTPATIENT ADHD TREATMENT STUDY "COMPLY"}

P. Wehmeier ${ }^{1}$, A. Schacht ${ }^{1}$, R.W. Dittmann ${ }^{1,2}$, T. Banaschewski $^{2}$

${ }^{1}$ Medical Department, Lilly Deutschland GmbH, Bad Homburg, ${ }^{2}$ Central Institute of Mental Health, Mannheim, Germany

Aims: Patient-related factors and physicians' attitudes leading to the decision which particular treatment is prescribed for attentiondeficit/hyperactivity disorder (ADHD) have not been investigated in detail. We assessed the reasons for choice of medication and the socioeconomic status of treatment-naïve ADHD patients when initiated on ADHD medication.

Method: Multicenter, prospective, 6-month observational study in children and adolescents with ADHD (ICD-10 or DSM-IV). Demographic, disease, and socioeconomic characteristics, and ADHD-related symptoms (ADHD-RS, CGI-S, GIPD) were captured at baseline, and compared between two medication groups (non-stimulant vs. stimulant). Physicians' reasons for choice of medication were recorded. Correlations between those reasons were explored by Pearson's correlation coefficient, Cohen's Kappa, and cluster analyses.

Results: Overall, 504 patients, mean age 9.6 years (range 6-17), 27.4\% female, were included. Non-stimulant medication was prescribed in $50.0 \%$ and stimulant in $49.0 \%$ of patients (1\% received both). In the non-stimulant group, patients were significantly older ( 0.6 years), had significantly more problems with regard to their psychosocial situation, and had more concomitant psychiatric disorders. There were no significant differences in questionnaire scores. Physicians' reasons for prescribing medication differed for non-stimulant vs. stimulant treatment: duration of action $(73.4 \% / 28.7 \%)$, beneficial for compliance $(60.3 \% / 40.1 \%)$, and patient/parent decision (33.7\%/26.3\%) were reasons in favor of non-stimulant treatment; good efficacy $(93.1 \% / 73.0 \%)$, good tolerability $(66.4 \% / 44.1 \%)$, and well-priced $(38.1 \% / 2.8 \%)$ in favor of stimulant treatment.

Conclusion: Patients initiating stimulant or non-stimulant ADHD treatment differed significantly on some points according to their baseline characteristics. Physicians' reasons for choice of medication clearly differed between the two medication groups. 